\title{
INVESTIGATION OF THE FREQUENCY OF CEREBRAL TRANSVERSE VENOUS SINUS STENOSIS IN IDIOPATHIC INTRACRANIAL HYPERTENSION
}

\author{
Fatma Yilmaz Can'1, Mehlika Panpalli Ates ${ }^{1}$
} ${ }^{1}$ University of Health Sciences, Diskapi Yildirim Beyazit Training and Research Hospital, Department of Neurology, Ankara,
Turkey.

Address for Correspondence: Mehlika Panpalli Ates, E-mail: muefhulkika@gmail.com

Received: 06.06.2021; Accepted: 20.08.2021; Available Online Date: 20.09.2021

(C) Copyright 2021 by Dokuz Eylül University, Institute of Health Sciences - Available online at https://dergipark.org.tr/en/pub/jbachs

Cite this article as: Panpalli-Ates M, Yilmaz-Can F. Investigation of the Frequency of Cerebral Transverse Venous Sinus Stenosis in Idiopathic Intracranial Hypertension. J Basic Clin Health Sci 2021; 3: 132- 136.

\begin{abstract}
Purpose: Idiopathic intracranial hypertension $(\mathrm{IIH})$ is an increase in intracranial pressure with a normal cerebrospinal fluid (CSF) composition that is not due to a secondary cause. The existence of cerebral transverse venous sinus stenosis and changes in venous outflow in IIH has recently gotten a lot of attention, and this situation is becoming increasingly important in terms of diagnosis and treatment plan.

This study aimed to investigate how frequent cerebral transverse venous sinus stenosis is in patients with IIH.

Methods: The demographic profile of 27 patients with IIH who were followed up in the hospital's neurological headache outpatient clinic and the occurrence of cerebral transverse venous stenosis on cranial magnetic resonance venography (MRV) were studied. Considering the pre-diagnosis of cerebral venous thrombosis (SVT), patients who underwent magnetic resonance venography (MRV) and whose SVT was ruled out during their follow-up were included as the control group. This control group consisted of 48 patients diagnosed with migraine, tension-type headache (TTH), and new-onset daily persistent headache.
\end{abstract}

Results: When MRVs were investigated, cerebral transverse venous sinus stenosis was detected in $\% 55.6(n=15)$ of IIH patients and 25\% $(n=12)$ of the control group $(p=0.017)$.

Conclusions: The frequency of cerebral transverse sinus stenosis in MRV of patients diagnosed with $\mathrm{IIH}$ was found to be significantly higher in this study than in the control group. These findings indicated that cerebral transverse venous stenosis can play a role in the progression of IIH.

Keywords: Cerebral venous sinus stenosis, idiopathic intracranial hypertension, headache, magnetic resonance venography

\section{INTRODUCTION}

Idiopathic intracranial hypertension $(\mathrm{IIH})$ is an increase in intracranial pressure that occurs without a secondary cause and in which the cerebrospinal fluid (CSF) composition is normal. It is a disease of uncertain etiology that affects children and the elderly, even though that it is primarily a disease of obese women of reproductive age [1].

The $\mathrm{IIH}$ is a progressive headache that mostly develops subacutely. It can begin abruptly and acutely at times. Although intracranial pressure improves during follow-up of patients with $\mathrm{IH}$, 
headaches may persist and relapse is common. It is a persistent, progressive headache that typically spreads to the entire head, increases with intracranial pressure-increasing maneuvers such as coughing and stretching, and may be accompanied by morning nausea and vomiting and is non-pulsatile [1,2]. It is similar to TTH in that it affects the entire head and is progressive and persistent. It has been reported that half of the patients may have allodynia, and the pain of patients with allodynia resembles migraine [3]. Having no papilledema is uncommon. Patients without papilledema have a clinical image close to chronic new-onset daily persistent headache or migrainous headache, which response to antimigraine treatment $[2,3]$.

The modified Dandy criteria are used to make the diagnosis. Neuroimaging findings are used in the absence of papilloedema and abducens nerve palsy, according to these criteria. The existence of cerebral transverse venous sinus stenosis, an empty sella appearance, flattening of the posterior globe, and expansion of the perioptic subarachnoid space (with or without tortuous optic nerve) are these findings. At least three of these criteria should be met for IIH to be suspected [4,5]. In a recent review article, sensitivity and specificity were calculated for each of these parameters. The highest sensitivity and specificity values $(97 \%$ and $93 \%)$ were found in cerebral transverse venous sinus stenosis [6].

The majority of $\mathrm{IIH}$ patients have venous sinus pathologies [7-11]. The most important point in distinguishing, however, is the exclusion of sinus vein thrombosis.

This study aimed to look into the frequency of cerebral transverse venous sinus stenosis in patients who had IIH.

\section{MATERIAL-METHOD}

Patient group; It was made up of 27 patients who visited the Neurology headache outpatient clinic between 2017 and 2020 and were diagnosed with IIH using the Modified Dandy criteria [3,4]. Control group; It included 48 patients, during follow-up, whose cerebral MRVs were performed to rule out cerebral vein thrombosis based on clinical suspicion of headache symptoms; based on the International Classification of Headache Disorders III-beta criteria (ICHD III-beta) diagnosed to tension-type headache, migraine, and new-onset daily persistent headache [12].
Patients with a history of brain structural disease, existing degenerative brain disease, use of medications that affect the central nervous system were removed from the research. Patients with cerebral vein thrombosis and those whose definitive diagnosis could not be made during their follow-up were removed from the study.

Magnetic resonance venography: Cerebral MRVs were examined during the diagnosis to rule out cerebral venous sinus thrombosis. On the basis of their the cerebral venography of patients, transverse venous sinus stenosis was determined. According to the scoring system developed by Farb et al. in patients, cerebral transverse venous sinus stenosis was defined [8]. Every patient in the study had their demographic characteristics, headache characteristics, MR venography, and follow-up files examined.

\section{Statistical analysis}

Statistical analysis was carried out using a Statistical Package for Social Sciences (SPSS ${ }^{\circledR}$ ) 22.0 for Windows and Mac os $x$. Shapiro-Wilk test was used to test the hypothesis of normality. Numeric data were presented as mean \pm standard deviation, median and nominal data were presented as percentages (\%). Student t-test was used for the statistical analysis within and between the groups in the age. Two main correlation coefficients (pearson and spearman) were used to determine the degree of relationship between two variables. $p$ values less than 0.05 were accepted as statistically significant.

\section{RESULTS}

The study included $27 \mathrm{IIH}$ patients and 48 control patients with similar demographic characteristics who were followed up with a diagnosis of $\mathrm{IIH}$ according to the modified Dandy criteria $[4,5]$. The mean age of IIH patients was $32.9 \pm 9.17$ (20-50, median: 32$)$, while the control group was $36.2 \pm 9.56$ (20-52, median: 36.5) $(p=0.293)$. There were 26 female IIH patients $(96.3 \%)$ and one male IIH patient $(3.7 \%)$. The control group consisted of $40(83.3 \%)$ females and $8(16.7 \%)$ males $(p=0.094)$ (Table 1$)$.

Complaints of all patients included in the study; were headaches spreading to the whole head, lasting at least 5 consecutive days, waking from sleep moderate to severe (Visual Analogue Scale- VAS between 7-10). 
Table 1. Demographic characteristics of $\mathrm{IIH}$ and control groups

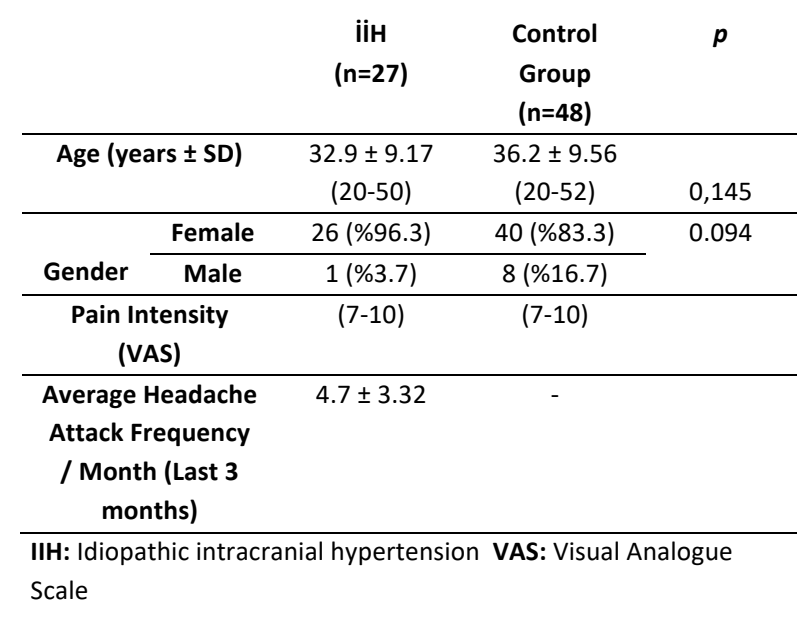

The neurological examinations of the patients in the control group were normal. $90.9 \%$ of them had normal brain MRIs, while four had chronic ischemic-gliotic changes.

CSF pressure at the diagnostic stage of IIH patients was found to be $284.7 \pm 44.22$ (200-350).

There is no difference in BMI or weight difference between patients with (respectively; $30.6 \pm 6.02$; $81 \pm$ 18.43), and without (respectively; $30.1 \pm 8.43 ; 77 \pm$ 17.92) transverse sinus vein stenosis in $\mathrm{IIH}$ patients (respectively; $p=0.867, p=0.576$ ).

The presence of cerebral transverse venous sinus stenosis was observed in $14(51.9 \%)$ of $\mathrm{IIH}$ patients and $12(25 \%)$ of the control group ( $p=0.036)$. Ten of the 27 patients with $\mathrm{IIH}$ had cerebral transverse venous sinus stenosis on the left, three on the right, and one was bilateral, while 11 of the 40 patients in the control group had it on the left and one on the right (Table 2).

Table 2. Comparison of Cerebral Venous Sinus Stenosis between $\mathrm{IIH}$ and Control Groups

\begin{tabular}{llll}
\hline & $\begin{array}{l}\text { iiH } \\
(\mathbf{n = 2 7 )}\end{array}$ & $\begin{array}{l}\text { Control } \\
\text { Group } \\
(\mathbf{n = 4 8 )}\end{array}$ & $p$ \\
\hline $\begin{array}{l}\text { Transverse venous sinus } \\
\text { stenosis }\end{array}$ & $15(\% 55.6)$ & $12(\% 25)$ & $\mathbf{0 . 0 1 7}$ \\
\hline $\begin{array}{l}\text { Right Transverse venous } \\
\text { sinus stenosis }\end{array}$ & $4(\% 14.9)$ & $1(\% 2.1)$ & \\
\hline $\begin{array}{l}\text { Left transverse venous } \\
\text { sinus stenosis }\end{array}$ & $10(\% 37)$ & $11(\% 22.9)$ & \\
\hline $\begin{array}{l}\text { Bilateral Transverse } \\
\text { venous sinus stenosis }\end{array}$ & $1(\% 3.7)$ & 0 \\
\hline
\end{tabular}

IIH: Idiopathic intracranial hypertension
In the control group, 14 patients were diagnosed with migraine, 32 with TTH, and ten with new-onset daily persistent headache.

When the presence of pulse-tinnitus was questioned in patients with $\mathrm{IIH}$, a moderate correlation was found in patients with cerebral transverse venous sinus stenosis in their MRV $(p=0.002$, Rho: +0.562$)$.

\section{DISCUSSION}

This study demonstrated that the frequency of transverse sinus stenosis in the cerebral venographies of patients with $\mathrm{IH}$ was significantly higher than in the control group, which consisted of $\mathrm{TTH}$, migraine, and new-onset daily persistent headache according to ICHD III-beta diagnostic criteria [12].

Despite the fact that IIH has been known for a long time, the pathogenesis of the disease remains unknown. Increased cerebral blood volume, excessive CSF production, venous outflow obstruction, and impaired CSF resorption are some of the proposed mechanisms [1]. In recent years, it has been proposed that inflammatory factors can also play a role [11]. In addition, dysregulation of fluid homeostasis, endocrinopathies, and white matter oedema due to fluid and electrolyte imbalances have been proposed as etiologic factors [14]. Furthermore, the high incidence of $\mathrm{IIH}$ in women of reproductive age with a high BMI supports the theory that metabolic or hormonal factors, and adipose tissue, may play a role in the pathogenesis [1,2]. The lack of additional endocrinological disorder and gender differences between the groups in our study indicated that cerebral transverse venous sinus stenosis played a role in pathogenesis as well.

There is increasing evidence that $\mathrm{IH}$ patients are particularly associated with cerebral transverse venous sinus stenosis. The incidence of stenosis in cerebral venographies of patients with a prior diagnosis of $\mathrm{IH}$ was reported to be greater than in normal healthy people, and cerebral transverse sinus stenosis was reported in more than half of patients with $\mathrm{IH}[9,15]$. In our study, $51.9 \%$ of patients with $\mathrm{IIH}$ detected cortical transverse sinus stenosis.

Furthermore, cerebral transverse sinus stenosis was determined to be statistically substantially higher in IHD patients than in the control group $(p=0.036)$. There has never been a comparison of the frequency of cerebral transverse sinus stenosis in $\mathrm{IIH}$ and other headaches like migraine, TTH, and new everyday chronic headache. This condition is probably 
considered a natural variant or structural hypoplasia unless intracranial pressure is elevated, and it does not appear to be remarkable. The question of whether these patients will develop $\mathrm{IIH}$ in the future is important.

Increased intracranial pressure may be caused by a disruption between CSF outflow circulation and venous sinus pressure (ICP). For example, in the case of sinus venous thrombosis, ICP increases secondary to increased sinus venous pressure. A pressure gradient across the narrowed segment may be correlated with any stenotic condition. The reversibility of stenosis after ICP normalization suggests a direct and dynamic relationship between ICP and venous sinus appearance [7]. In IIH patients, high intracranial venous sinus pressure has been observed $[1,7,11,16]$. In a previous study of $\mathrm{IIH}$ patients who were treated medically for an average of 6.2 years, it was discovered that while CSF opening pressure and symptoms improved, cerebral transverse venous sinus stenosis persisted, leading to the conclusion that cerebral transverse venous sinus stenosis may exist prior to the increase in intracranial pressure and symptoms of $\mathrm{IIH}$, and that venous stenosis is insufficient to increase intracranial pressure [17]. However, cerebral transverse sinus stenosis was not evaluated here by measuring the degree of stenosis before and after treatment. Another research found no correlation between the degree of unilateral cerebral transverse venous sinus stenosis and the clinic of IIH [18].

Pulse-synchronous (pulsatile) tinnitus, on the other hand, is known to be present in more than half of $\mathrm{IIH}$ patients. It has been stated that stenting of cerebral transverse venous sinus stenosis in $\mathrm{IIH}$ patients with pulsatile tinnitus can often improve these complaints $[1,19]$.

In our research, patients with IIH had a substantially higher incidence of cerebral transverse sinus stenosis than the control group with TTH, migraine, or newonset daily persistent headaches. This led us to think that cerebral transverse venous sinus stenosis may play an important role in the pathophysiology of $\mathrm{IIH}$; that there is a relationship between cerebral transverse venous sinus stenosis and IIH disease. However, it is still uncertain whether increased intracranial pressure causes cerebral transverse venous sinus stenosis in $\mathrm{IIH}$ or whether $\mathrm{IIH}$ is caused by cerebral transverse venous sinus stenosis. Prospective studies are needed on this subject.

\section{CONCLUSION}

The pathogenesis of IIH is still unknown, despite the fact that it has been known and treated for many years. There is no single etiologically reliable evidence supporting the pathophysiology. The incidence of cerebral transverse venous stenosis in patients with $\mathrm{IIH}$ was found to be higher than other headache clinical features such as migraine, TTH, and new-onset daily persistent headache in this study.

Acknowledgements: This paper was not funded by anyone and the author declared that it has received no financial support. The author declares no conflict of interest related to this work.

Conflict of Interest: None.

Ethical approval: The study was carried out according to the 1964 Helsinki Declaration and was approved by the Institutional Local Ethics Committee (2020-84/10).

Informed Consent: For the study, written and verbal consent was obtained from the patients.

Financial Disclosure: None

\section{REFERENCES}

1. Thurtell MJ. Idiopathic Intracranial Hypertension. Continuum (Minneap Minn). 2019; 25(5): 12891309.

2. Baykan B, Ekizoğlu E, Altıokka Uzun G. An update on the pathophysiology of idiopathic intracranial hypertension alias pseudotumorcerebri. Agri. 2015; 27(2): 63-72.

3. Ekizoglu E, Baykan B, Orhan EK, Ertas M. The analysis of allodynia in patients with idiopathic intracranial hypertension. Cephalalgia 2012; 32(14): 1049-1058.

4. De Simone R, Ranieri A, Montella S, Friedman DI, Liu GT, Digre KB. Revised diagnostic criteria for the pseudotumorcerebri syndrome in adults and children. Neurology. 2014; 82: 1011-1012.

5. Friedman DI, Liu GT, Digre KB. Revised diagnostic criteria for the pseudotumorcerebri syndrome in adults and children. Neurology 2013; 81: 1159-1165.

6. Zur D, Anconina R, Kesler A, Lublinsky S, Toledano R, Shelef I. Quantitative imaging biomarkers for dural sinüs patterns in idiopathic intracranial hypertension. Brain Behav. 2017; 7(2): e00613.

7. Bono F, Giliberto C, Mastrandrea C, et al. Transverse sinus stenoses persist after normalization of the CSF pressure in $\mathrm{IIH}$. Neurology. 2005; 65(7): 1090-1093.

8. Farb RI, Vanek I, Scott JN, et al. Idiopathic intracranial hypertension: the prevalence and 
morphology of sinovenous stenosis. Neurology. 2003; 60: 1418-1424.

9. Chan W, Neufeld A, Maxner C, Shankar J. Irreversibility of transverse venous sinüs stenosis and optic nerve edema post-lumbar puncture in idiopathic intracranial hypertension. Can J Ophthalmol. 2019; 54(2): e57-e59.

10. Horev A, Hallevy H, Plakht Y, Shorer Z, Wirguin I, Shelef I. Changes in cerebral venous sinuses diameter after lumbar puncture in idiopathic intracranial hypertension: a prospective MRI study. J Neuroimaging. 2013; 23: 375-378.

11. Rohr A, Bindeballe J, Riedel C, et al. The entire dural sinus tree is compressed in patients with idiopathic intracranial hypertension: a longitudinal, volumetric magnetic resonance imaging study. Neuroradiology 2012; 54: 25-33.

12. Headache Classification Committee of the International Headache Society (IHS). The International Classification of Headache Disorders, 3rd edition (beta version). Cephalalgia. 2013; 33(9): 629-808.

13. Sinclair AJ, Ball AK, Burdon MA, et al. Exploring the pathogenesis of $\mathrm{IIH}$ : an inflammatory perspective. J Neuroimmunol 2008; 201-2: 212220

14. Gideon P, Sørensen PS, Thomsen C, Ståhlberg $F$, Gjerris F, Henriksen O. Increased brain water self-diffusion in patients with idiopathic intracranial hypertension. AJNR Am J Neuroradiol 1995; 16(2): 381-387.

15. Morris PP, Black DF, Port J, Campeau N. Transverse Sinus Stenosis Is the Most Sensitive MR Imaging Correlate of Idiopathic Intracranial Hypertension. AJNR Am J Neuroradiol. 2017; 38(3): 471-477.

16. Bidot S, Bruce BB, Saindane AM, Newman NJ, Biousse V. Asymmetric papilledema in idiopathic intracranial hypertension. J Neuroophthalmol. 2015; 35(1): 31-36.

17. Bono F, Quattrone A. Clinical course of idiopathic intracranial hypertension with transverse sinüs stenosis. Neurology. 2013; 81(7): 695.

18. Riggeal BD, Bruce BB, Saindane AM, et al. Clinical course of idiopathic intracranial hypertension with transverse sinus stenosis. Neurology. 2013; 80(3): 289-95.

19. Boddu S, Dinkin M, Suurna M, Hannsgen K, Bui $X$, Patsalides A. Resolution of Pulsatile Tinnitus after Venous Sinus Stenting in Patients with
Idiopathic Intracranial Hypertension. PLoS One. 2016; 11(10): e0164466. 